Weiss, C. F., Glazko, A. J., and Weston, J. K. (1960). Chloramphenicol in the newborn infant. A physiologic explanation of its toxicity when given in excessive doses. New England fournal of Medicine, 262, 787.

A. W. Craft, ${ }^{\star}$ J. T. Brocklebank, E. N. HeY, and R. H. JACKSON

Department of Child Health, Royal Victoria Infirmary, Newcastle upon Tyne NE1 4LP.

^Correspondence to Dr. A. W. Craft.

\section{Effect of head rotation on jugular vein blood flow}

During a cardiac investigation blood oxygen saturations in the innominate veins were noticed to vary when the child moved his head. It seemed

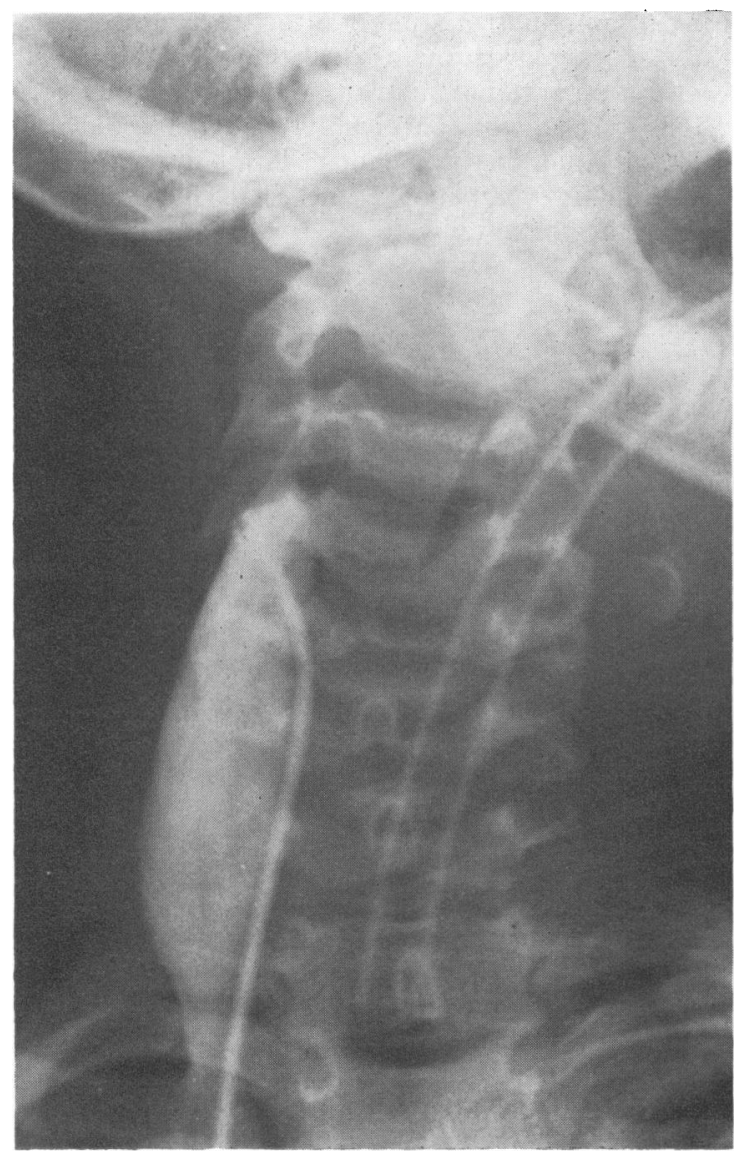

(a) possible that alterations in jugular vein flow were responsible, particularly in view of the well-known changes in cervical venous hums caused by rotation of the head. To study this effect, internal jugular venography was performed by manual injections of radio-opaque medium under fluoroscopy at the beginning of cardiac catheterization in 60 infants and children who had various congenital cardiac anomalies but no other major malformations.

\section{Method}

A catheter introduced from the leg was passed into one or both of the internal jugular veins where this could be done with a minimum of manipulation and without inconvenience to the child. The head was then rotated to varying degrees, as far as the child could maintain by himself if unanaesthetized. 16 children were anaesthetized for the cardiac investigation, and the others were sedated with trimeprazine, except for small infants who received no sedation. Though the 60 patients represented only a minority of the total number undergoing investigation, it is unlikely that the results were affected.

\section{Results}

Flow in an internal jugular vein was not affected by contralateral rotation of the head (Fig. 1a), but

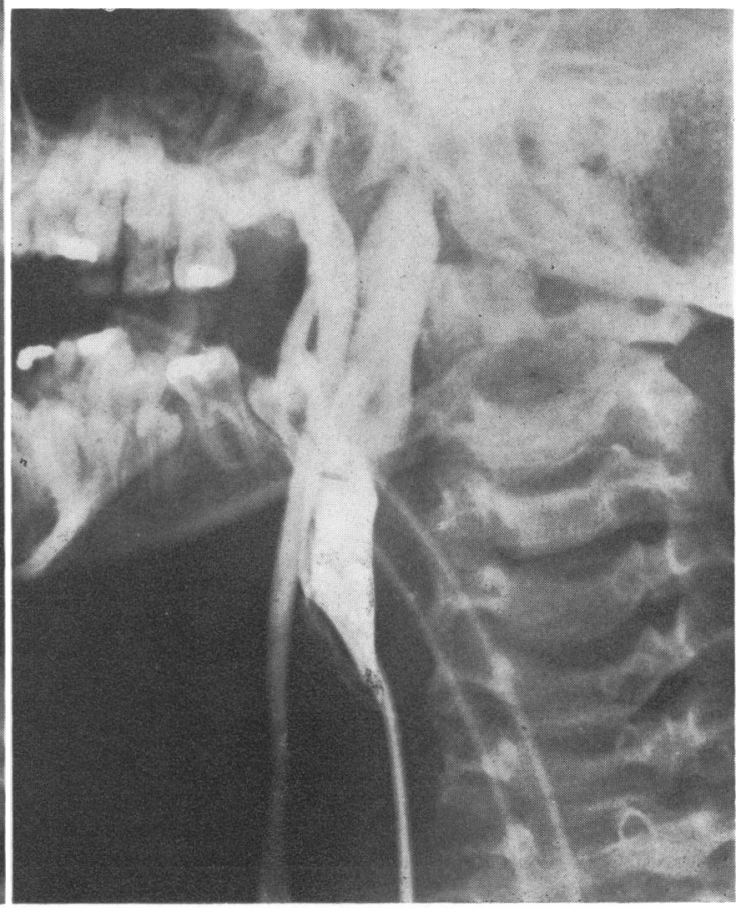

(b)

FIG. 1.-Right internal jugular venogram. (a) Head turned to left showing free downward flow, (b) head turned to right showing vein occluded; escape of medium to smaller veins. 
ipsilateral rotation by $45^{\circ}$ or more caused narrowing of the middle third of the vein, presumably by soft tissues related to the sternomastoid muscle. Ipsilateral rotation of the head to almost $90^{\circ}$ caused complete occlusion of the vein in 41 children (Fig. 1b), in all 16 children who were anaesthetized, and in 25 of the remaining 44 . In 24 of these 41 children only $75^{\circ}$ rotation was needed, and in 15 of them only $60^{\circ}$ rotation, to interrupt downward flow in the vein completely. Withdrawal of the catheter just after an injection did not affect the obstruction.

Sedation other than anaesthesia, age, and diagnosis were not factors in determining the production of obstruction. In the minority of children where both internal jugular veins were entered, ipsilateral head rotation usually affected each vein similarly.

In some cases medium injected into the upper part of an internal jugular vein occluded by head rotation escaped entirely into smaller veins, but more commonly some medium flowed retrogradely into the lateral sinus. It then sometimes disappeared rapidly, but in about half the children with an occluded vein the medium remained visible in the lateral sinus for 1 to 3 minutes and sometimes longer, even though by this time no medium could be seen outside the skull (Fig. 2). Downward flow in a vein obstructed by head rotation was not restored by digital occlusion of the opposite internal jugular vein, though the face sometimes became suffused.

Mean pressure gradients between the upper and lower thirds of the internal jugular veins, normally 0 to $\frac{1}{2} \mathrm{mmHg}$, rose to 1 to $3 \frac{1}{2} \mathrm{~mm}$ during occlusion.

Effect on venous oxygen saturations. Blood oxygen saturations in the internal jugular, innominate, and superior caval veins are usually similar during a cardiac investigation; the higher saturations in tributaries such as the subclavian veins, often 80 to $85 \%$ in asymptomatic children, are outweighed by the large cerebral flow, usually at 60 to $70 \%$ saturation. In 5 cases rotation of the head to one side caused the ipsilateral innominate vein saturation to rise by over $25 \%$, well outside the usual sampling variation of 5 to $10 \%$. Presumably this occurred through deviation of the lowersaturation internal jugular flow to the other side. This effect might conceivably raise the suspicion of partial pulmonary venous flow into the left innominate vein should the head happen to be turned to the left.

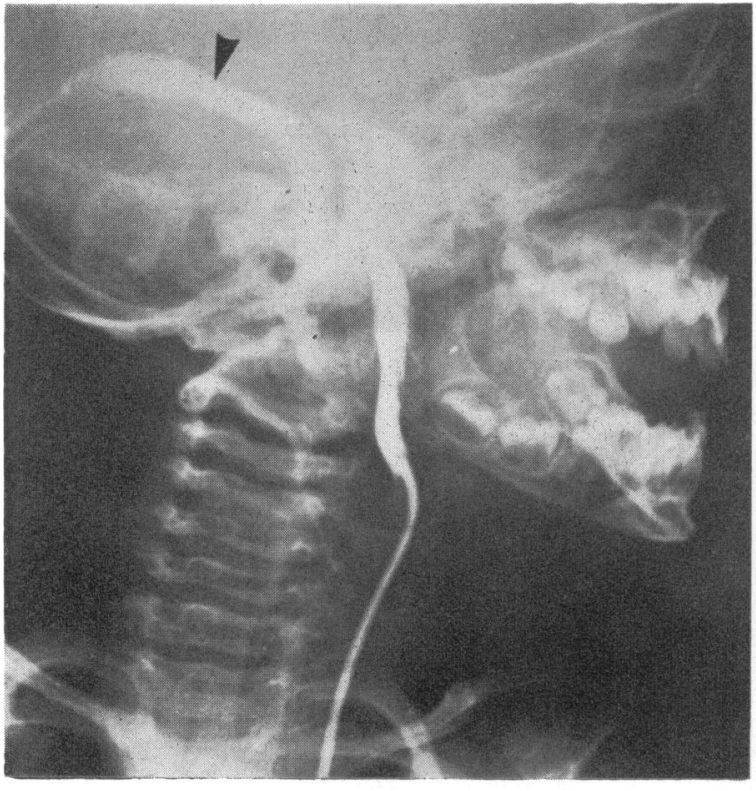

(a)

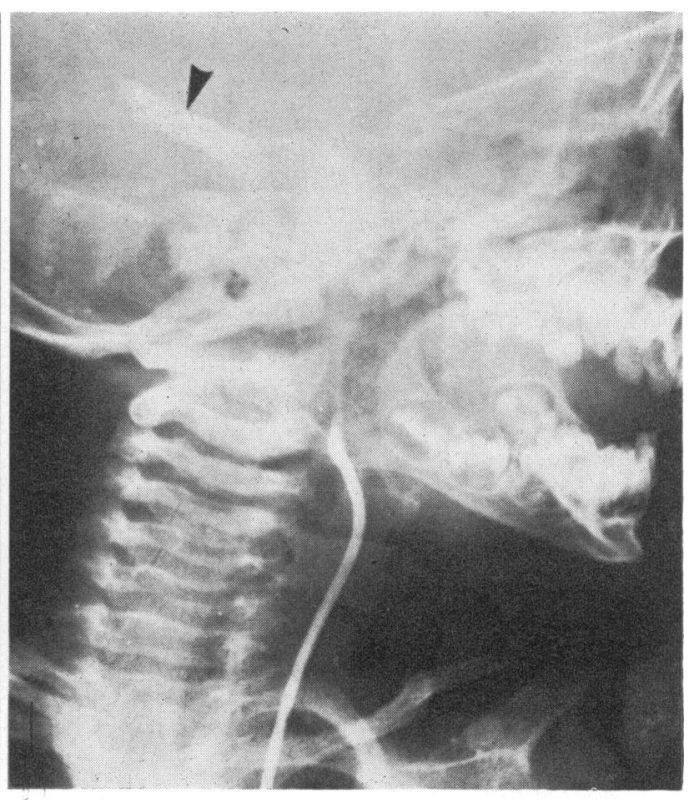

(b)

FIG. 2.-Left internal jugular venogram. (a) Head turned to left showing retrograde filling of left lateral sinus (arrow), (b) 3 minutes later, medium still present in left lateral sinus. 


\section{Comment}

No references to similar observations have been found, though neurosurgeons are aware of the effect of unusual head positions on cerebral congestion at operation. The observations may also be relevant in the following situations.

(1) In children undergoing manipulations involving an internal jugular vein, since the head is often turned fully to the other side.

(2) In children who already have occlusion of one of their internal jugular veins (more particularly if its upper tributaries are involved).

(3) In the management of children with cyanotic heart lesions or infective lesions predisposing to thrombosis (if stasis is indeed a further provocative factor).
(4) In the management of hydrocephalus and of possible intracranial haemorrhage.

\section{Summary}

Rotation of the head to one side causes obstruction of the ipsilateral internal jugular vein in most anaesthetized and many nonanaesthetized children, and may cause intracranial venous stasis.

G. H. WATSON

Royal Manchester Children's Hospital, and the Department of Child Health, University of Manchester.

^Correspondence to G.H.W., Royal Manchester Children's Hospital, Pendlebury, Manchester M27 1HA. 